\title{
Irrversibility of entanglement manipulations: Vagueness of the entanglement of cost and entanglement of distillation
}

\author{
Won-Young Hwang* and Keiji Matsumoto ${ }^{\dagger}$ \\ IMAI Quantum Computation and Information Project, ERATO, Japan Science and Technology Corporation, Daini Hongo \\ White Building 201, 5-28-3, Hongo, Bunkyo, Tokyo 133-0033, Japan
}

We show that the entanglement of cost and entanglement of distillation can be vague when we consider a more general form of entanglement manipulation in which we collectively deal with not only states of our concern but also other states. We introduce the most general entanglement manipulation in which the formation and distillation can be simultaneously performed. We show that in a certain case entanglement manipulations are reversible with respect to the most general entanglement manipulation. This broadens our scope of vision on the irreversibility of entanglement manipulations.

03.67.-a

Entanglement is a key ingrediant in quantum information processing. Without full entanglement, we cannot expect the exponential speedup in quantum computation over the classical one in the case of pure states [1]. In quantum communications like superdense coding [2] and quantum teleportation [3], entanglement is indispensible. At the same time the entanglement is a valuable resource that should not be wasted, since entanglement can only be obtained by (costly) non-local operations. For the better understanding and manipulation of the entangled states, we should classify them as well as possible. Quantification of the entanglement, namely the measure of entanglement is, therefore, a central issue in quantum information theory $[4,5]$.

Entanglement of cost $E_{C}$ and entanglement of distillation $E_{D}$ were proposed by Bennett et al. [4]. These are an important pair of measures because they are intuivitely motivated. On the other hand, irreversibility in entanglememt manipulations is another important issue. The question is that 'Can we distill as much entanglement from a state $\rho$ as we have consumed for the formation of it, with only quantum local operations with classical communications (LOCCs)?'. If this is the case, we can reverse the formation process by the distillation process without loss of entanglement. Then we can say that the entanglement manipulation is reversible. In finite-copies case, entanglement manipulation is shown to be irreversible both for pure [6-8] and mixed states [5]. However, in asymptotic case where infinitely many identical copies are dealt with, entanglement manipulation of the pure states is shown to be reversible, namely we have $E_{C}(\rho)=E_{D}(\rho)$ for pure states [9]. However, it has been conjectured that the entanglement manipulation is irreversible for mixed states even in the asymptotic case. Indeed it has been shown to be case in a few examples [10-12]. It is often said that the irreversiblity in the asymptotic manipulation implies a genuine irreversibility [13]. Discovery of a new genuine irreversiblity has a large implication on natural sciences. However, the irreversibility depends on how general the entanglement manipulation that we consider is. In the asymptotic manipulation, we only deal with infinitely many copies of a state that we are concerned about. That is, we do not consider other states. However, due to (possible) nonadditivity of entanglement, we need to consider a more general form of entanglement manipulation in which we collectively deal with both the states of our concern and other states. We will see how the generalization broadens our scope of vision on irreversibility of mixed state entanglement manipulation [10-12]. For example, we find that it is reversible with respect to a general entanglement manipulation in a certain case.

This paper is organized as follows. First, we introduce the entanglement of cost, entanglement of distillation, and the irreversibility. Next, we show that we should consider collective-formation and collective-distillation in the case of mixed state. With respect to these, the entanglement of cost and entanglement of distillation can be vague. Next, we consider a more general entanglement manipulation, that of 'the center of entanglement'. We discuss the irreversibility with respect to the center of entanglement; The formation and distillation processes are reversible with respect to the center of entanglement in a certain case.

The measure of entanglement for the bipartite pure state $|\psi\rangle\langle\psi|$ is given by

$$
E(|\psi\rangle\langle\psi|)=S\left(\operatorname{Tr}_{B}|\psi\rangle\langle\psi|\right),
$$

where $S(\rho)$ is the von Neumann entropy of $\rho$, i.e., $S(\rho)=$ $-\operatorname{Tr}\left(\rho \log _{2} \rho\right)$ and $B$ denotes the second party of two parties Alice and Bob [9].

Entanglement of cost $E_{C}$ of a state $\rho$ is inf. of $N / M$ in the limit of large $N$, when we create the state by an (approximate) transformation like

$$
\left|\Psi^{-}\right\rangle\left\langle\left.\Psi^{-}\right|^{\otimes N} \rightarrow \rho^{\otimes M}\right.
$$

with LOCCs. Here $N, M$ are integers and $\left|\Psi^{-}\right\rangle=$ $(1 / \sqrt{2})\left(|0\rangle_{A}|1\rangle_{B}-|1\rangle_{A}|0\rangle_{B}\right)$ is one of the Bell states. Entanglement of distillation $E_{D}$ of a state $\rho$ is sup. of $N^{\prime} / M$ in the limit of large $N^{\prime}$, when we distill the state $\rho$ by an (approximate) transformation like

$$
\rho^{\otimes M} \rightarrow\left|\Psi^{-}\right\rangle\left\langle\left.\Psi^{-}\right|^{\otimes N^{\prime}}\right.
$$


with LOCCs. (For more rigorous definitions see Refs. $[10,14]$.)

The irreversibility problem is whether it can be $N=$ $N^{\prime}$ in a manipulation

$$
\left|\Psi^{-}\right\rangle\left\langle\left.\Psi^{-}\right|^{\otimes N} \rightarrow \rho^{\otimes M} \rightarrow \mid \Psi^{-}\right\rangle\left\langle\left.\Psi^{-}\right|^{\otimes N^{\prime}} .\right.
$$

In the case of finite numbers $\left(N, N^{\prime}\right.$, and $\left.M\right)$ of copies, it is irreversible or $N>N^{\prime}$ [5]. In the asymptotic case where infinitely many copies are considered, however, due to (possible) non-additivity of entanglement, it might be reversible. Recently, Vidal and Cirac [10,11] and Matsumoto et al. [12] have shown that the asymptotic entanglement manipulation is irreversible, that is, $E_{C}(\rho)>E_{D}(\rho)$, for certain states $\rho$ 's. However, the irreversibility of other general states is still an open problem.

However, it is due to collective-manipulation that entanglement manipulation with finite copies can be different from that with infinite copies, the asymptotic case. Collective-manipulation is considered in the asymptotic manipulation of the pure states $[9,15]$ : Entanglement manipulation of a single copy of pure states is highly irreversible [6-8]. That is, except for local unitary operations, we lose certain amount of entanglement during the manipulation. However, if we collectively manipulate infinitely many copies of pure states, the amount of the lost entanglement is negligible. In other words, the more general manipulation we use, the less entanglement we lose.

In the same way, collective-formation like

$$
\left|\Psi^{-}\right\rangle\left\langle\left.\Psi^{-}\right|^{\otimes N} \rightarrow \rho_{1}^{\otimes M_{1}} \otimes \rho_{2}^{\otimes M_{2}} \otimes \cdots\right.
$$

and collective-distillation like

$$
\rho_{1}^{\otimes M_{1}} \otimes \rho_{2}^{\otimes M_{2}} \otimes \cdots \rightarrow\left|\Psi^{-}\right\rangle\left\langle\left.\Psi^{-}\right|^{\otimes N^{\prime}} .\right.
$$

can be more advantageous than entanglement manipulation where each state $\rho_{i}(i=1,2,3, \ldots)$ is separately formated and distilled, respectively. For example, in a collective-formation $\left|\Psi^{-}\right\rangle\left\langle\left.\Psi^{-}\right|^{\otimes N} \rightarrow \rho_{1}^{\otimes M_{1}} \otimes \rho_{2}^{\otimes M_{2}}\right.$, it can be that $N<M_{1} E_{C}\left(\rho_{1}\right)+M_{2} E_{C}\left(\rho_{2}\right)$. Here $E_{C}$ is the entanglement of cost that is defined with respect to separate entanglement manipulations $\left|\Psi^{-}\right\rangle\left\langle\left.\Psi^{-}\right|^{\otimes n} \rightarrow \rho_{j}^{\otimes m}\right.$ $(j=1,2)$. However, in these cases we can see that there is no unique way of assigning entanglement of cost or entanglement of distillation to each state $\rho_{i}$. In other words, entanglement of creation and entanglement of distillation are vague, respectively, when we deal with collectiveformation and collective-distillation of many copies of states that include not only a state whose entanglement of cost and entangement of distillation we want to define but also other different states. In collective manipulation where we deal with many copies of a state whose entanglement of cost and entanglement distillation we want to define, there is no such vagueness problem. There is a unique solution, that is, to equally assign entanglement to each state [9]. A search for a solution for how to distribute the entanglement of cost and entanglement of distillation to different states is worthwhile. It can be that a solution will be given by a certain kind of self-consistency condition. The irreversibility problem now is whether we can close the loop of the transformations in Eq. (5) and (6), that is, whether $N=N^{\prime}$. This problem can be different from that of $\left|\Psi^{-}\right\rangle\left\langle\left.\Psi^{-}\right|^{\otimes N} \rightarrow \rho_{i}^{\otimes M} \rightarrow \mid \Psi^{-}\right\rangle\left\langle\left.\Psi^{-}\right|^{\otimes N^{\prime}}\right.$.

Now let us consider the most general entanglement manipulation, namely that of 'banks of entanglement'. Let us assume that a pair of banks of entanglement were established on the Earth and Mars. A pair of customers, say $\mathrm{E}_{1}$ and $\mathrm{M}_{1}$, who are respectively on the Earth and Mars, will make an order for banks to do certain desired manipulations on their pairs of qubits at each bank. Using the banks is advantageous because collective manipulations can possibly save entanglement, as we have discussed. The more orders the banks gather before they perform a big collective manipulation, the more entanglement they can save. Moreover, the banks can adopt all possible form of collective manipulations like the catalytic manipulation [16], Morikoshi's [17], catalytic manipulation with unlimited amount of bound entanglement [18-20], and the asymptotic generalization of catalytic manipulation [21]. In fact, concept of the banks has been implicit in previous discussions. But there was a restriction that the formation and distillation are separately performed. However, the banks of entanglement do not have to keep the restriction. More general transformation that the banks perform is

$$
\begin{aligned}
\left|\Psi^{-}\right\rangle\left\langle\left.\Psi^{-}\right|^{\otimes N} \otimes \rho_{1}^{\otimes M_{1}} \otimes \rho_{2}^{\otimes M_{2}} \otimes \cdots\right. & \\
& \rightarrow \rho_{1}^{\prime \otimes M_{1}} \otimes \rho_{2}^{\prime \otimes M_{2}} \otimes \cdots \otimes\left|\Psi^{-}\right\rangle\left\langle\left.\Psi^{-}\right|^{\otimes N^{\prime}}\right.
\end{aligned}
$$

where formation and distillation are performed simultaneously.

Now let us consider the irreversibility problem. A certain customer will give the banks an order for formation of a state $\rho_{1}$ from the Bell states. The other will give the banks an order for distillation of the Bell state from another state $\rho_{2}$. Assuming a lot of customers who have different orders, we can introduce distribution functions $P_{i}^{f}$ and $P_{i}^{d}$ for each state $\rho_{i}(i=1,2,3, \ldots)$. Here $P_{i}^{f}$ and $P_{i}^{d}$ are frequencies that formation and distillation of a state $\rho_{i}$ is ordered, respectively. Let us consider a case in which $P_{i}^{f}=P_{i}^{d}$. Here what the banks have to do is only to appropriately re-distribute the qubits to the customers. Thus it is obviously reversible. It is not that we can always get the best case. However, even when the distribution functions are not the same but only partially overlap, the amount of lost entanglement will be decreased.

In conclusion, the irreversibility in entanglement manipulations is dependent upon how general manipulation we deal with. We need to consider a more general form of entanglement manipulation in which we collectively deal with not only states of our concern but also other states. With respect to the general manipulation, the entanglement of cost $E_{C}$ and distillation $E_{D}$ can be vague. We introduced the most general entanglement manipulation, namely that of the banks of entanglement, in which the 
formation and distillation do not have to be separatedly performed. We showed that in a certain case entanglement manipulations are reversible with respect to the most general entanglement manipulation. This broadens our scope of vision on the irreversibility of entanglement manipulations.

\section{ACKNOWLEDGMENTS}

We are very grateful to Prof. Hiroshi Imai and Japan Science and Technology Corporation for financial sup-

ports. We are also very grateful to Dr. G. Vidal for discussions.

* Present address: Department of Electrical and Computer Engineering, Northwestern University, Evanston, IL 60208, USA; Email: wyhwang@ece.northwestern.edu

† Email: keiji@qci.jst.go.jp

[1] R. Jozsa, in ERATO workshop on Quantum Information Science 2001. (http://www.qci.jst.go.jp/eqis/)

[2] C.H. Bennett and S.J. Wiesner, Phys. Rev. Lett. 69, 2881 (1992).

[3] C.H. Bennett, G. Brassard, C. Crepeau, R. Jozsa, A. Peres, and W.K. Wootters, Phys. Rev. Lett. 70, 1895 (1993).

[4] C.H. Bennett, D.P. Divincenzo, J.A. Smolin, and W.K. Wootters, Phys. Rev. A 54, 3824 (1996).

[5] V. Vederal and M.B. Plenio, Phys. Rev. A 57, 1619 (1998).

[6] H.-K. Lo and S. Popescu, Phys. Rev. A 63, 022301 (2001).

[7] M.A. Nielsen, Phys. Rev. Lett. 83, 436 (1999).

[8] G. Vidal, Phys. Rev. Lett. 83, 1046 (1999).

[9] C.H. Bennett, H.J. Berstein, S. Popescu, and B. Schmacher, Phys. Rev. A 53, 2046 (1996).

[10] G. Vidal and J.I. Cirac, Phys. Rev. Lett. 86, 5803 (2001).

[11] G. Vidal and J.I. Cirac, Phys. Rev. A 65, 012323 (2002).

[12] K. Matsumoto, T. Shimono, and A. Winter, quant$\mathrm{ph} / 0206148$.

[13] M. Horodecki, P. Horodecki, and R. Horodecki, Phys. Rev. Lett. 84, 4260 (2000).

[14] P.M. Hayden, M. Horodecki, and B.M. Terhal, J. Phys. A 34, 6891 (2001).

[15] S. Popescu and D. Rorhlich, Phys. Rev. A 56, 3319 (1997).

[16] D. Jonathan and M.B. Plenio, Phys. Rev. Lett. 83, 3566 (1999).

[17] F. Morikoshi, Phys. Rev. Lett. 84, 3189 (2000).

[18] E.M. Rains, Phys. Rev. A 60, 173 (1999).

[19] E.M. Rains, Phys. Rev. A 60, 179 (1999).

[20] V. Vederal, Phys. Lett. A 262, 121 (1999).

[21] V. Vederal and E. Kashefi, Phys. Rev. Lett. 89, 037903 (2002). 